\title{
Avaliação da atenção ao parto normal em um hospital do Sul do Brasil
}

\section{Evaluation of normal delivery care in a hospital in Southern Brazil}

\author{
Sâmela Basi Fagundes \\ Universidade Estadual de Ponta Grossa - UEPG - Ponta Grossa - Brasil \\ basisamela@gmail.com \\ Ana Claudia Garabeli Cavalli Kluthcovsky \\ Universidade Estadual de Ponta Grossa - UEPG - Ponta Grossa - Brasil \\ anafabio2009@gmail.com \\ Ana Paula Ditzel \\ Universidade Estadual de Ponta Grossa - UEPG - Ponta Grossa - Brasil \\ prof.go.anapaula@gmail.com
}

\begin{abstract}
Resumo
O objetivo desse estudo é avaliar as práticas da atenção hospitalar durante o trabalho de parto e parto normal em um hospital universitário no Sul do Brasil, com ênfase nos procedimentos obstétricos. Trata-se de um estudo descritivo, transversal e de abordagem quantitativa, realizado com 140 puérperas pós-parto normal. Foram coletados dados socioeconômicos, obstétricos e do recém-nato, mediante entrevistas com as puérperas e consulta ao prontuário médico e ao Cartão da Gestante. Os resultados são apresentados por frequências absoluta e relativa e medidas estatísticas descritivas. A média de idade das parturientes foi de 24,9 anos ( $D P=5,6)$, sendo a maioria branca $(66,4 \%)$ e com companheiro $(89,3 \%)$. As práticas consideradas úteis foram: dieta oral $(75,7 \%)$, liberdade de posição e movimento (88,6\%), métodos não farmacológicos para aliviar a dor $(87,1 \%)$, acompanhante no pré-parto (95\%) e parto $(79,3 \%)$ e partograma $(86,4 \%)$. As práticas consideradas prejudiciais foram: posição supina $(87,1 \%)$ e manobra de Kristeller $(6,4 \%)$, além de enema e tricotomia (práticas não realizadas nas puérperas deste estudo). As práticas usadas inapropriadamente foram: amniotomia $(4,3 \%)$, ocitocina $(49,3 \%)$, episiotomia $(10,7 \%)$ e analgesia (0\%). Enfatiza-se a importância da assistência ao trabalho de parto e parto com o mínimo de intervenções compatíveis com o que é preconizado.
\end{abstract}

Palavras-chave: parto obstétrico, saúde materno-infantil, pesquisa sobre serviços de saúde.

\section{Abstract}

The objective of this study is to evaluate the practices of hospital care during labor and vaginal delivery, in a university hospital in the South of Brazil. A descriptive, crosssectional, quantitative study developed with 140 puerperal postpartum normal. We collected socioeconomic, obstetrical and neonatal data through interviews with postpartum women, medical records and Pregnancy Card. Absolute and relative frequencies and statistical descriptive measures presented the results. The mean age of parturients was 
24.9 years $(S D=5.6)$, most of them were white $(66.4 \%)$ and had partners $(89.3 \%)$. The useful practices included oral diet (75.7\%), freedom of position and movement $(88.6 \%)$, non-pharmacological methods to alleviate pain (87.1\%), companion on prepartum (95\%) and parturition (79.3\%) and partogram (86.4\%). Harmful practices included supine position $(87.1 \%)$ and Kristeller maneuver $(6.4 \%)$. The use of enemy and trichotomy was not prescribed for any parturient. Practices inappropriately used included: amniotomy (4.3\%), oxytocin (49.3\%), episiotomy (10.7\%) and analgesia (0.0\%). This study showed the importance of assistance to labor and delivery with minimum of interventions.

Keywords: obstetric delivery, maternal and child health, health services research.

\section{Introdução}

A assistência ao parto passa por um momento de transição no Brasil e no mundo. O que vinha sendo visto como um evento médico e que demandava intervenções, atualmente tem como foco o protagonismo da mulher e sua autonomia, que ganhou visibilidade com o movimento da humanização (SOUSA et al., 2016).

A Organização Mundial de Saúde (OMS), preocupada com o cenário mundial de excesso de intervenções no parto, publicou em 1996 orientações técnicas para o cuidado de parturientes saudáveis. Mais de duas décadas depois, em 2018, essas recomendações foram atualizadas devido às mudanças ocorridas no panorama global dos serviços de maternidade. Segundo a OMS o objetivo da assistência ao parto se expandiu e, além de evitar a morbidade e mortalidade materna e neonatal, as novas intervenções são fundamentais para garantir que o parto seja uma experiência positiva para as mulheres e suas famílias. Ainda destaca como os cuidados centrados na mulher podem otimizar a qualidade da assistência ao parto e nascimento através de uma abordagem holística baseada nos direitos humanos (WORLD HEALTH ORGANIZATION, 2018).

$\mathrm{Na}$ área da saúde, a avaliação é uma ferramenta importante para o planejamento e a gestão dos sistemas e serviços, produzindo informações e conhecimentos para aferir a efetividade das intervenções, o uso adequado dos recursos e satisfazer os usuários do sistema. Assim, a avaliação aprimora teorias e também fornece subsídios às intervenções necessárias (SANCHO; DAIN, 2012).

Nesse contexto, a avaliação da qualidade do atendimento ao parto é muito importante, pois, a partir da avaliação é possível verificar se as práticas em saúde estão de acordo com as evidências científicas disponíveis e o seu impacto na rotina dos serviços em saúde (SANDIN-BOJÖ et al., 2012). Assim, baseado na proposta de humanização, o desenvolvimento da prática obstétrica baseada em evidências prevê atitudes e comportamentos dos profissionais da saúde que contribuam com um olhar para a integralidade da assistência obstétrica (NAGAHAMA; SANTIAGO, 2008).

Considerando a importância da qualidade na assistência à saúde da parturiente e o escasso número de publicações sobre o tema, esse estudo teve por objetivos avaliar as práticas da atenção hospitalar durante o trabalho de parto e parto normal na maternidade de um hospital universitário no Sul do Brasil, com ênfase nos procedimentos obstétricos.

\section{Material e Métodos}

Foi realizado um estudo descritivo, transversal e de abordagem quantitativa na maternidade de um hospital universitário localizado na Região Sul do Brasil.

O tamanho da amostra foi calculado com base em uma estimativa de existência de partograma em $90 \%$ dos prontuários, precisão relativa de $6 \%$ e nível de significância de $5 \%$, resultando uma amostra de no mínimo 96 partos normais. Utilizou-se a prevalência do achado de partograma no prontuário por ser um dos indicadores preconizados pela 
OMS para avaliação da qualidade de assistência ao parto normal (GIGLIO; FRANÇA; LAMOUNIER, 2011). Neste estudo, em função da amostra ser não-probabilística, optouse por trabalhar com uma amostra maior que o mínimo necessário, totalizando-se 140 pacientes puérperas, a fim de aumentar a precisão dos resultados.

Para a coleta de dados, inicialmente era realizada uma consulta ao prontuário médico, buscando todas as pacientes admitidas como internação para parto. Entre todas as gestantes admitidas, foram selecionadas aquelas que tiveram parto normal. Foram incluídas no estudo todas as puérperas que tiveram parto normal classificadas como risco habitual no Cartão da Gestante, com 18 anos de idade ou mais e que concordaram em participar do estudo assinando o Termo de Consentimento Informado Livre e Esclarecido. Foram excluídas puérperas submetidas à cesariana, aquelas classificadas como risco moderado ou alto no Cartão da Gestante, e que tiveram óbito fetal.

Os dados foram obtidos por meio de entrevistas com as puérperas durante o período de internação, até 48 horas após o parto, entre dezembro de 2017 e abril de 2018. Essas entrevistas foram realizadas com a parturiente no leito, de forma padronizada pela mesma entrevistadora, em um único encontro. Além da entrevista com a puérpera foram coletados dados do Cartão da Gestante e do prontuário médico.

Foram coletados dados socioeconômicos, obstétricos e do recém-nato. Os dados socioeconômicos foram: idade, estado civil, cor de pele, escolaridade, trabalho remunerado e renda familiar mensal per capita. Os dados obstétricos e do recém-nato foram: número de consultas de pré-natal, número de gestações, intercorrências na gravidez, idade gestacional, tempo de internação até o parto em horas, peso do recémnato em gramas e o Índice de Apgar (APGAR, 1953), que avalia clinicamente o recémnato, segundo sua Aparência, Pulso, Gesticulação, Atividade e Respiração, ao primeiro e quinto minuto após o nascimento.

Para avaliar a qualidade da assistência ao parto normal, foi utilizada uma classificação das práticas obstétricas organizada em três categorias: práticas demonstradamente úteis e que devem ser encorajadas (liberdade de posição e movimento, métodos não farmacológicos para alívio da dor, uso do partograma, presença de acompanhante no pré-parto, presença de acompanhante no parto e oferecimento de dieta oral); práticas claramente prejudiciais ou ineficazes e que devem ser eliminadas (posição de litotomia durante o parto, manobra de Kristeller, enema e tricotomia) e práticas frequentemente utilizadas de modo inapropriado durante o trabalho de parto e parto (amniotomia, infusão de ocitocina, analgesia e episiotomia) (SOUSA et al., 2016).

As diferentes práticas da atenção ao parto normal foram analisadas para o total das parturientes. Os dados foram processados em microcomputador, no banco de dados Excel. As variáveis foram analisadas utilizando-se frequências absoluta e relativa e medidas estatísticas descritivas com o auxílio do programa SPSS (Statistical Package for Social Sciences), versão 15.0.

O projeto foi aprovado pela Comissão de Ética em Pesquisa (CAAE: 79581317.1.0000.0105, parecer CEP 2.409.711), nos termos da Resolução 466/2012 do Conselho Nacional de Saúde.

\section{Resultados}

As parturientes apresentaram idade média de 24,9 anos ( $D P=5,6)$, sendo que os demais dados socioeconômicos, obstétricos e do recém-nato estão apresentados na Tabela 1. Para o total das parturientes, a grande maioria apresentava entre 20 e 34 anos de idade $(77,1 \%)$ e tinha companheiro estável $(89,3 \%)$. A maioria se autodeclarou da cor branca $(66,4 \%)$ e tinha mais de oito anos de estudo $(68,6 \%)$. Pouco mais da metade relatou ter trabalho remunerado $(65,7 \%)$ e a maioria declarou renda familiar mensal per capita inferior a um salário-mínimo $(73,2 \%)$. 
Tabela 1: Variáveis socioeconômicas, obstétricas e do recém-nato de puérperas de um hospital universitário no Sul do Brasil, 2017-2018 ( $n=140)$

\begin{tabular}{|c|c|c|}
\hline Variáveis & $\mathbf{n}$ & $\%$ \\
\hline \multicolumn{3}{|l|}{ Idade em anos } \\
\hline Menos de 20 & 20 & 14,3 \\
\hline 20 a 34 & 108 & 77,1 \\
\hline 35 ou mais & 12 & 8,6 \\
\hline \multicolumn{3}{|l|}{ Estado civil } \\
\hline Sem companheiro & 15 & 10,7 \\
\hline Com companheiro & 125 & 89,3 \\
\hline \multicolumn{3}{|l|}{ Cor da pele } \\
\hline Não branca & 47 & 33,6 \\
\hline Branca & 93 & 66,4 \\
\hline \multicolumn{3}{|l|}{ Escolaridade } \\
\hline Até 8 anos de estudo & 44 & 31,4 \\
\hline Mais de 8 anos de estudo & 96 & 68,6 \\
\hline \multicolumn{3}{|l|}{ Trabalho remunerado } \\
\hline Não & 48 & 34,3 \\
\hline Sim & 92 & 65,7 \\
\hline \multicolumn{3}{|c|}{ Renda familiar mensal per capita em reais } \\
\hline Até 1 salário mínimo & 90 & 73,2 \\
\hline Mais de 1 salário mínimo & 33 & 26,8 \\
\hline \multicolumn{3}{|l|}{ Início do pré-natal } \\
\hline$\leq 12$ semanas & 105 & 75,0 \\
\hline$>12$ semanas & 35 & 25,0 \\
\hline \multicolumn{3}{|l|}{ Número de consultas de pré-natal } \\
\hline$\leq 6$ & 21 & 15,0 \\
\hline 7 ou mais & 119 & 85,0 \\
\hline \multicolumn{3}{|l|}{ Intercorrências na gravidez } \\
\hline Sim & 4 & 2,9 \\
\hline Não & 136 & 97,1 \\
\hline \multicolumn{3}{|l|}{ Primeira gestação } \\
\hline Sim & 44 & 31,4 \\
\hline Não & 56 & 68,6 \\
\hline \multicolumn{3}{|c|}{ Tempo de trabalho de parto hospitalar em horas } \\
\hline$<8$ & 127 & 92,0 \\
\hline 8 ou mais & 11 & 8,0 \\
\hline \multicolumn{3}{|l|}{ Complicação no parto } \\
\hline Sim & 6 & 4,3 \\
\hline Não & 134 & 95,7 \\
\hline \multicolumn{3}{|l|}{ Idade gestacional } \\
\hline$<37$ semanas & 6 & 4,3 \\
\hline 37 semanas ou mais & 134 & 95,7 \\
\hline \multicolumn{3}{|l|}{ Peso ao nascer em gramas } \\
\hline$<2.500$ & 4 & 3,0 \\
\hline 2.500 a 3.999 & 123 & 93,2 \\
\hline 4.000 ou mais & 5 & 3,8 \\
\hline \multicolumn{3}{|l|}{ Apgar no primeiro minuto } \\
\hline$\leq 7$ & 15 & 10,9 \\
\hline 8 ou mais & 123 & 89,1 \\
\hline \multicolumn{3}{|l|}{ Apgar no quinto minuto } \\
\hline$\leq 7$ & 1 & 0,7 \\
\hline 8 ou mais & 138 & 99,3 \\
\hline
\end{tabular}

Sem informação ou a parturiente não soube informar: Renda familiar mensal per capita em reais: $n=17(12,1 \%)$, Tempo de trabalho de parto hospitalar em horas: $n=2(1,4 \%)$, Peso ao nascer em gramas: $n=8(5,7 \%)$, Apgar no primeiro minuto: $n=2(1,4 \%)$, Apgar no primeiro minuto: $n=1(0,7 \%)$.

Fonte: Autores (2018) 
No que diz respeito à gestação, a maioria das mulheres eram multíparas (dois partos ou mais) $(68,6 \%)$ e realizaram sete ou mais consultas de pré-natal $(85,0 \%)$, sendo que apenas 2,9\% delas apresentaram algum tipo de intercorrência durante a gestação, sendo elas doença hipertensiva específica da gestação, diabetes mellitus gestacional e sangramentos vaginais não especificados.

Ao internamento, quase todas as parturientes (95,7\%) apresentavam gestação a termo (37 semanas de gestação ou mais) sendo a média da idade gestacional na admissão de $39,2(\mathrm{DP}=1,4)$ semanas. O tempo de trabalho de parto hospitalar foi de até oito horas em $92,0 \%$ da amostra. Houve complicações em 4,2\% dos partos, sendo um caso de hemorragia intraparto, um caso de hipotonia uterina, dois casos de fórcipe, um caso de retenção placentária e uma inversão uterina.

Foram observados baixos percentuais de prematuridade $(4,3 \%)$, de baixo peso ao nascer (3\%) e de Apgar menor que sete no quinto minuto $(0,7 \%)$. A maioria dos recémnatos apresentava peso adequado ao nascer (entre 2.500 e 3.999 gramas) (93,2\%), sendo a média do peso ao nascimento, em gramas, de 3.313,7 (DP=397,7). Os valores de Apgar no primeiro e quinto minuto foram iguais ou maiores a oito em $89,1 \%$ e $99,3 \%$ dos recém-natos, respectivamente.

A Tabela 2 apresenta a ocorrência de práticas demonstradamente úteis durante o trabalho de parto e parto para o total das puérperas. A maioria delas teve liberdade de posição e movimento durante o trabalho de parto $(88,6 \%)$ e realizou algum método não farmacológico para alívio da dor como massagem e banhos $(87,1 \%)$. Também na maioria dos prontuários médicos $(86,4 \%)$, o partograma estava presente e completamente preenchido, e em 13,6\% estavam preenchidos de modo incompleto ou não constavam no prontuário. Quase a totalidade das pacientes teve seu acompanhante de escolha durante o trabalho de parto (95\%). Porém, um número menor de puérperas relatou a presença de acompanhante durante o parto $(79,3 \%)$, comparado ao percentual de acompanhante no trabalho de parto. O percentual de puérperas que relatou receber dieta oral foi de $75,7 \%$.

Tabela 2: Frequência das práticas demonstradamente úteis durante o trabalho de parto e parto normal em parturientes de um hospital universitário no Sul do Brasil, 2017-2018 ( $n=140)$

\begin{tabular}{lcc}
\hline Variáveis & $\mathbf{n}$ & $\%$ \\
\hline Liberdade de posição e movimento & 124 & 88,6 \\
$\quad$ Sim & 16 & 11,4 \\
Não & 122 & 87,1 \\
Métodos não farmacológicos para alívio da dor & 18 & 12,3 \\
$\quad$ Sim & 121 & 86,4 \\
$\quad$ Não & 19 & 13,6 \\
Uso do partograma & & \\
$\quad$ Sim & 155 & 95,0 \\
Não & 7 & 5,0 \\
Presença de acompanhante (pré-parto) & 111 & 79,3 \\
$\quad$ Sim & 29 & 20,7 \\
Não & & 75,7 \\
Presença de acompanhante (parto) & 106 & 24,3 \\
$\quad$ Sim & 34 & \\
Não & & \\
Oferecimento de dieta oral & & \\
Sim & & \\
Não & &
\end{tabular}

Fonte: Autores (2018)

Quanto às frequências das práticas consideradas prejudiciais ou ineficazes durante o trabalho de parto e parto e que devem ser abandonadas, observou-se que a posição de litotomia ou posição supina foi utilizada de rotina durante o parto e foi referida por $87,1 \%$ das puérperas. A manobra de Kristeller foi utilizada em $6,4 \%$ dos partos, já o enema e 
tricotomia não foram usados em nenhuma parturiente (Tabela 3).

Tabela 3: Frequência das práticas claramente prejudiciais ou ineficazes durante o trabalho de parto e parto normal em parturientes de um hospital universitário no Sul do Brasil, 2017-2018 $(n=140)$

\begin{tabular}{lcc}
\hline Variáveis & $\mathbf{n}$ & $\%$ \\
\hline Posição de litotomia ou supina durante o parto & & \\
Sim & 122 & 87,1 \\
Não & 18 & 12,9 \\
Manobra de Kristeller & 9 & 6,4 \\
$\quad$ Sim & 131 & 93,6 \\
Não & 0 & 0,0 \\
Enema & 140 & 100,0 \\
Sim & & 0,0 \\
Não & 0 & 100,0 \\
Tricotomia & 140 & \\
Sim & &
\end{tabular}

Fonte: Autores (2018)

A Tabela 4 apresenta a frequência das práticas que frequentemente são utilizadas de modo inapropriado durante o trabalho de parto e parto normal em parturientes.

Tabela 4: Frequência das práticas que frequentemente são utilizadas de modo inapropriado durante o trabalho de parto e parto normal em parturientes de um hospital universitário no Sul do Brasil, 2017-2018 ( $n=140)$

\begin{tabular}{lcc}
\hline Variáveis & $\mathbf{n}$ & $\%$ \\
\hline Amniotomia & & 4,3 \\
Sim & 6 & 95,7 \\
Não & 134 & 49,3 \\
Infusão de ocitocina & 69 & 50,7 \\
Sim & 71 & 0,0 \\
Não & & 100,0 \\
Analgesia & 0 & 10,7 \\
Sim & 140 & 89,3 \\
Não & 15 & 125 \\
Episiotomia & & \\
Sim & & \\
Não & & \\
\hline
\end{tabular}

Fonte: Autores (2018)

Em relação às práticas obstétricas que frequentemente são usadas de modo inapropriado durante o trabalho de parto e parto, a amniotomia foi relatada em apenas $4,3 \%$ dos prontuários. A infusão de ocitocina, no primeiro ou segundo períodos do trabalho de parto ocorreu em $49,3 \%$ dos partos analisados. O uso de medicamentos uterotônicos no manejo ativo do terceiro período de trabalho de parto é realizado de rotina na maternidade estudada e todas as puérperas receberam a medicação. A analgesia de parto não foi utilizada em nenhuma paciente. Na maioria das mulheres a episiotomia não foi realizada $(89,3 \%)$ (Tabela 4$)$.

\section{Discussão}

Neste estudo, as 140 puérperas entrevistadas apresentaram média de idade de $24,9$ anos ( $D P=5,6)$, com predomínio na faixa etária de 20 a 34 anos $(77,1 \%)$. Valor semelhante à média de idade de 24,8 anos, encontrado em um estudo realizado em duas 
maternidades públicas municipais de assistência a 314 partos de risco habitual situadas na cidade de Natal (CARVALHO; BRITO, 2016) e em 23,6\% de 991 parturientes de um centro de parto normal em São Paulo (LOBO et al., 2010).

Quase $90 \%$ das puérperas relataram ter companheiro, diferente de estudo com 404 mulheres que tiveram partos normais em 13 hospitais de Goiânia, onde 76,5\% apresentavam união consensual (GIGLIO; FRANÇA; LAMOUNIER, 2011), bem de outros onde 58,4\% (LOBO et al., 2010) tinham companheiro e quase $80 \%$ se declararam solteiras (CARVALHO; BRITO, 2016). A maioria das puérperas que declarou ter mais de oito anos de estudo (68,6\%), um pouco menor que os $75,4 \%$ do estudo realizado em São Paulo (LOBO et al., 2010). Trabalho remunerado foi relatado por 65,7\% das parturientes, assim como no estudo de Dias et al., (2016) com (73,33\%) e muito diferente de outros em que a maioria das puérperas declarou não trabalhar (CARVALHO; BRITO, 2016; LOBO et al., 2010).

O acompanhamento pré-natal foi realizado por $100 \%$ das puérperas deste estudo, resultado semelhante ao da literatura (CARVALHO; BRITO, 2016; DIAS et al., 2016).

Quanto às características do recém-nato, no presente estudo, as porcentagens de variáveis adequadas como gestações a termo, peso adequado e Apgar no quinto minuto de oito ou mais foram superiores a $90 \%$. Já as variáveis neonatais não adequadas como idade gestacional menor do que 37 semanas, peso ao nascer inferior a 2500 gramas e Apgar no quinto minuto de sete ou menos tiveram baixa porcentagem, evidenciando resultados neonatais satisfatórios na maternidade em questão e corroborando dados da literatura (GIGLIO; FRANÇA; LAMOUNIER, 2011; CARVALHO; BRITO, 2016).

$\mathrm{Na}$ avaliação da qualidade da atenção ao trabalho de parto e parto normal, dentre as práticas demonstradamente úteis e que devem ser encorajadas está a liberdade de posição e movimento, a qual foi relatada por $88,6 \%$ das puérperas, resultado próximo ao de $96,0 \%$ encontrado em um estudo realizado em Belo Horizonte que avaliou a assistência ao parto em instituições de saúde onde atuam, conjuntamente médicos e enfermeiras obstétricas (SOUSA et al., 2016). Em recente pesquisa que sobre a assistência ao parto em dois maternidades públicas no Rio de Janeiro, em 2.914 partos assistidos por enfermeiras obstétricas, 55,45\% das parturientes foram estimuladas à deambulação (VARGENS; SILVA; PROGIANTI, 2017). Outro estudo, que avaliou as intervenções obstétricas durante o trabalho de parto e parto em mulheres brasileiras de risco habitual, demonstrou um número menor, em que apenas $44,3 \%$ das parturientes tiveram liberdade de movimento (LEAL, et al., 2014). A mobilidade adequada da parturiente traz benefícios positivos no trabalho de parto, que incluem a maior tolerância à dor, o que evita uso de fármacos, também melhorando a evolução da dilatação e reduzindo a duração da fase ativa do trabalho de parto (BIO; BITTAR; ZUGAIB, 2006).

Outra prática com alto percentual de relato foi a oferta de métodos não farmacológicos para alívio da dor, relatada por $87,1 \%$ das puérperas, valor maior que 0 encontrado no estudo de Sousa e colaboradores (2016), de 74,2\% e de Novo e colaboradores (banho de aspersão em 23,48\% e massagem em 34,80\%) (NOVO et al., 2016). Isso pode demonstrar apoio por parte da equipe hospitalar em tomar medidas para tornar o trabalho de parto mais confortável para as pacientes. De modo semelhante às práticas encontradas na literatura (LOBO et al., 2010), a maioria das puérperas deste estudo referiu realização de banhos de aspersão em água quente, deambulação e massagens, práticas recomendadas pelo Ministério da Saúde (BRASIL, 2016).

A presença do partograma em $86,4 \%$ dos prontuários está acima da média descrita na literatura, de 77,4\% (SOUSA et al., 2016), 62\% (NOVO et al., 2016), 28,5\% (GIGLIO; FRANÇA; LAMOUNIER, 2011) e de 2,2\% (CARVALHO; BRITO, 2016). Por outro lado, foi observado presença de partograma em 98,5\% dos 406 prontuários avaliados em três maternidades de risco habitual de Curitiba (OLIVEIRA et al., 2015). Há evidência que a utilização do partograma pode ser benéfica e linhas de ação mais precoces que uma linha 
de ação de quatro horas aumenta as possibilidades de intervenções sem benefícios para a mãe ou a criança (BRASIL, 2016). Porém, é importante avaliar se o preenchimento do partograma corresponde às reais avaliações e intervenções realizadas.

Ter um acompanhante familiar no parto é um direito garantido no Brasil pela Lei no 11.108/2005, regulamentada pelo Ministério da Saúde pela Portaria 2418/2005 (BRASIL, 2005). A presença de acompanhante traz à parturiente maior grau de satisfação, além de estar relacionada a trabalhos de partos mais curtos e normais e menor uso de analgesai (BRÜGGEMANN; PARPINELLI; OSIS, 2005). Neste estudo, foi avaliada a presença de acompanhante durante o período de pré-parto e no parto. Observou-se uma maior frequência de acompanhante no período de pré-parto, de $95 \%$, enquanto $79,3 \%$ dos partos se deram com acompanhante, sendo essa redução por escolha da parturiente ou do próprio acompanhante. Ainda assim, os dados encontrados foram muito superiores quando comparados à literatura, de 19,5\% (GIGLIO; FRANÇA; LAMOUNIER, 2011). Em uma maternidade de atenção secundária em Sorocaba, foram avaliadas 100 primíparas puérperas, que tiveram parto normal, sendo que $85 \%$ tiveram familiares presentes (NOVO et al., 2016). Contudo, esses números poderiam ser ainda maiores, como em estudos realizados em Natal (85,7\% com acompanhante no parto) (CARVALHO; BRITO, 2016), Belo Horizonte (95,4\% com acompanhante durante a internação) (SOUSA et al., 2016) e São Paulo (92,2\% com acompanhante durante todo o parto) (LOBO et al., 2010).

O oferecimento de dieta oral incluindo líquidos e sólidos foi referido por $75,7 \%$ das puérperas pesquisadas, resultado acima do encontrado em estudos semelhantes que apresentaram percentuais de 54,6\% (SOUSA et al., 2016) de dieta oral e dieta zero em 62,0\% (GIGLIO; FRANÇA; LAMOUNIER, 2011). Pacientes sem acesso à dieta oral tiveram seu trabalho de parto relacionado às incertezas de possíveis intervenções, como cesarianas de emergência, ou curto período de trabalho de parto hospitalar, porém a ausência total de dieta pode atrapalhar a evolução adequada do trabalho de parto. As parturientes devem receber uma dieta leve e ingerir líquidos, de preferência soluções isotônicas ao invés de somente água, desde que não estejam sob efeito de opioides ou não apresentem fatores de risco iminentes para anestesia geral (BRASIL, 2016).

Com relação às práticas claramente prejudiciais ou ineficazes e que devem ser eliminadas do trabalho de parto e parto, a posição supina durante o parto foi a que obteve o maior percentual, de $87,1 \%$. Alguns estudos mostraram resultados de $66,8 \%$ (SOUSA et al., 2016) para posição deitada e 91,7\% (LEAL, et al., 2014) para posição de litotomia e 100\% para posição supina (GIGLIO; FRANÇA; LAMOUNIER, 2011). A posição supina é culturalmente a mais aceita, tanto por profissionais quanto para as próprias pacientes (SOUSA et al., 2016). Contudo, em partos assistidos por enfermeiras obstétricas, em $65,51 \%$ dos casos foi adotada a posição vertical no momento do parto (VARGENS; SILVA; PROGIANTI, 2017).

O Ministério de Saúde orienta que a parturiente deve ser incentivada a adotar a posição que ela achar mais confortável, incluindo as posições de cócoras, lateral ou quatro apoios (BRASIL, 2016). Portanto, cabe ao profissional de saúde incentivar a adoção de posição não-litotômica, já que a literatura sugere vários possíveis benefícios da postura ereta para o parto, como pequena redução na duração do segundo estágio do trabalho de parto, redução das episiotomias e das taxas de parto assitidos (GUPTA; HOFMEYR; SMYTH, 2015), além de maior comodidade, favorecimento da movimentação das pacientes, além de reduzir o esforço expulsivo (GAYESKI; BRÜGGEMANN, 2009).

A manobra de Kristeller é uma prática preocupante pelo potencial dano que pode causar tanto para à mãe quanto para o bebê. As recomendações atuais desencorajam a utilização dessa manobra (WORLD HEALTH ORGANIZATION, 2018). No presente estudo, ela foi relatada por $6,4 \%$ das puérperas, semelhante à avaliação realizada em Belo Horizonte, na qual 9,3\% das puérperas relataram esta prática (SOUSA et al., 2016) e bem abaixo dos $36,1 \%$ no estudo realizado com brasileiras (LEAL, et al., 2014), 55,4\% em 
hospitais de Goiânia (GIGLIO; FRANÇA; LAMOUNIER, 2011) e 58\% em Sorocaba (NOVO et al., 2016).

Práticas claramente prejudiciais como realização de enema e tricotomia não foram relatadas nesse estudo, indicando a eliminação das mesmas durante a assistência ao parto na maternidade em questão, da mesma forma que outro estudo (SOUSA et al., 2016). Essas frequências são ideais e semelhantes aos $2 \%$ de realização da tricotomia $e$ 0\% para enema (NOVO et al., 2016). A Diretriz Nacional de Assistência ao Parto Normal, divulgada pelo Ministério da Saúde deixa claro que o enema e a tricotomia pubiana e perineal não devem ser realizadas de forma rotineira durante o trabalho de parto (BRASIL, 2016).

Dentre as práticas frequentemente utilizadas de modo inapropriado durante o trabalho de parto e parto está a infusão de ocitocina, observada em $49,3 \%$ no presente estudo. Ela pode auxiliar na correção de alterações da evolução do trabalho de parto a partir de determinada dilatação cervical, porém seu uso inadequado pode resultar em complicações materno-fetais (WORLD HEALTH ORGANIZATION, 2018). Estudos relataram o uso de ocitócitos em 41,7\% (SOUSA et al., 2016) 45,8\% (GIGLIO; FRANÇA; LAMOUNIER, 2011), e 87\% (NOVO et al., 2016) das parturientes. Esses resultados evidenciam um alto grau de interferência na evolução do parto e um modelo de atenção medicalizado. Isso pode estar associado a intervenções desnecessárias e muitas vezes prejudiciais. Segundo a Organização Mundial da Saúde o uso precoce da amniotomia e da ocitocina para prevenir o atraso no trabalho de parto não é recomendado (WORLD HEALTH ORGANIZATION, 2018).

Nesse estudo, em 10,7\% das pacientes realizou-se episiotomia, valor maior do que 8,4\% (SOUSA et al., 2016) e 5,24\% (VARGENS; SILVA; PROGIANTI, 2017) relatados na literatura. No extremo oposto, os percentuais encontrados em outros estudos foram de $25,7 \%$ (LOBO et al., 2010) e de 53,5\% (LEAL, et al., 2014) e, mais recentemente, $94 \%$ das pacientes foram submetidas à episiotomia de rotina, evidenciando o alto grau de interferência durante a expulsão fetal (NOVO et al., 2016). Atualmente, a recomendação da Diretriz Nacional de Assistência ao Parto Normal é que essa prática não seja realizada rotineiramente durante o parto vaginal espontâneo (BRASIL, 2016).

A prática de amniotomia foi descrita em apenas $4,3 \%$ dos prontuários, valor menor que o encontrado em outros estudos, de 67,1\% (SOUSA et al., 2016) e 62,6\% (LOBO et al., 2010). Segundo a Diretriz Nacional de Assistência ao Parto Normal, a amniotomia precoce, associada ou não à ocitocina, não deve ser realizada de rotina em mulheres em trabalho de parto que estejam progredindo bem, porém ela pode ser considerada diante da suspeita de falha de progresso no primeiro estágio do trabalho de parto se as membranas estiverem íntegras. Nestes casos, a amniotomia pode reduzir o tempo do trabalho de parto por cerca de uma hora e aumentar a intensidade e dor das contrações (BRASIL, 2016).

Neste estudo nenhuma puérpera recebeu analgesia regional. Estudos avaliando a frequência de analgesia regional durante o trabalho de parto apontaram valores de 7,7\% (GIGLIO; FRANÇA; LAMOUNIER, 2011), 14\% (SOUSA et al., 2016) e 33,9\% (LEAL, et al., 2014). Considerando a alta frequência de métodos alternativos para controle da dor encontrada neste estudo, não podemos relacionar a ausência do uso de anestésico à negligência com a dor da paciente. Contudo, talvez haja falta de informação da parturiente em relação a essa opção, visto que a solicitação materna por analgesia de parto compreenderia indicação suficiente para sua realização, independente da fase do parto e do grau de dilatação, desde que esclarecida e discutida com a gestante, enfatizando os efeitos adversos e possíveis complicações (BRASIL, 2016).

O delineamento de um modelo de assistência intraparto que seja adaptável aos contextos individuais de cada país, possibilita economias substanciais de custos através da redução de intervenções desnecessárias durante o trabalho de parto e parto. Além 
disso, a avaliação de processo através da análise de fatores sociais, obstétricos e neonatais fornece uma base sólida para a adoção e adaptação de recomendações para a prestação de cuidados centrados na pessoa, baseados em evidências (WORLD HEALTH ORGANIZATION, 2018).

Esse estudo apresentou limitações, como o fato de ter sido realizado em uma única instituição, o que não permite generalização dos resultados. Algumas informações foram coletas do prontuário e do Cartão da Gestante, que dependem da qualidade das anotações realizadas. Além disso, as informações fornecidas pelas entrevistadas poderiam estar sujeitas ao viés de memória, o que foi minimizado pela entrevista ter sido realizada até 48 horas após o parto. Apesar dessas limitações, os resultados podem contribuir para uma melhor compreensão das práticas de atendimento hospitalar durante o trabalho de parto e parto normal.

\section{Conclusão}

$\mathrm{Na}$ avaliação das três categorias de práticas da atenção hospitalar durante o trabalho de parto e parto normal, foram observados tanto percentuais considerados satisfatórios (alta frequência de todas as práticas demonstradamente úteis) como não satisfatórios, com a maior parte dos partos sendo em posição supina e tendo estímulos como a infusão de ocitocina.

Apesar do desafio de evitar intervenções desnecessárias nas práticas de atenção ao parto, vemos que uma conduta mais humanizada já é realidade em alguns hospitais. Assim, é importante que a equipe de saúde esteja atualizada e capacitada para colocar em prática as recomendações fundamentais para acolher a gestante em trabalho de parto. Otimizando a qualidade das práticas de atenção ao parto através de uma abordagem holística, levando em conta a medicina baseada em evidências e os direitos e autonomia da parturiente, será mais provável uma experiência positiva e um melhor resultado na assistência com um mínimo de intervenção, compatível com o que é preconizado.

Estudos futuros poderiam avaliar os resultados de estratégias voltadas à melhoria do atendimento durante o trabalho de parto e parto, incluindo modelo multidisciplinar de atendimento hospitalar, monitoramento do trabalho de parto e parto com rigor científico e a humanização na assistência.

\section{Referências}

APGAR V. A proposal for a new method of evaluation of the newborn infant. Current Researches in Anesthesia \& Analgesia, v. 32, n. 4, p. 260-267, 1953.

- Ministério da Saúde. Diretrizes nacionais de assistência ao parto normal: relatório de recomendação. Brasília: Ministério da Saúde, 2016. 381 p.

BIO, E; BITTAR, R. E.; ZUGAIB, M. Influência da mobilidade materna na duração da fase ativa do trabalho de parto. Revista Brasileira de Ginecologia e Obstetrícia, v. 28, n. 11, p. 671-679, 2006.

BRASIL. Lei n.11.108, de 7 de abril de 2005. Dispõe sobre o direito da parturiente à presença de acompanhante durante o trabalho de parto, parto e pós-parto imediato, no âmbito do SUS. Brasília. Disponível em: $<$ https://www2.camara.leg.br/legin/fed/lei/2005/lei-11108-7-abril-2005-536370publicacaooriginal-26874-pl.html>. Acesso em: 14 fev. 2020. 
BRÜGGEMANN, O. M.; PARPINELLI, M. A.; OSIS, M. J. D. Evidências sobre o suporte durante o trabalho de parto/parto: uma revisão da literatura. Cadernos de Saúde Pública, v. 21, n. 5, p. 1316-1327, 2005.

CARVALHO, I. S.; BRITO, R. S. Using the Bologna Score to acess normal delivery healthcare. Revista da Escola de Enfermagem da USP, v. 50, n. 5, p. 741-748, 2016.

DIAS, E. G.; MONÇÃO, P. R.; CERQUEIRA, N. C.; SOUZA, M. A. S. Assistência de Enfermagem no parto normal em um hospital público de Espinosa, Minas Gerais, sob a ótica da puérpera. Revista Interdisciplinar, v. 9, n. 2, p. 38-48, 2016.

GAYESKI, M.E.; BRÜGGEMANN, O. M. Percepções de puérperas sobre a vivência de parir na posição vertical e horizontal. Revista Latino-Americana de Enfermagem, v. 17, n. 2, p. 1-7, 2009.

GIGLIO, M. R. P.; FRANÇA, E.; LAMOUNIER, L. A. Avaliação da qualidade da assistência ao parto normal. Revista Brasileira de Ginecologia e Obstetrícia, v. 33, n. 10, p. 297304, 2011.

GUPTA, J. K.; HOFMEYR, G. J.; SMYTH, R. M.D. Position in the second stage of labour for women without epidural anaesthesia. Cochrane Database Systematic Review 2015. Disponível em: https://www.cochranelibrary.com/cdsr/doi/10.1002/14651858.CD00 2006.pub4/epdf/full. Acesso em: 25 mar. 2019.

LEAL, M. C. et al. Intervenções obstétricas durante o trabalho de parto e parto em mulheres brasileiras de risco habitual. Cadernos de Saúde Pública, v. 30, n. 1, p. 17-32, 2014.

LOBO, S. F.; OLIVEIRA, S. M. J. V.; SCHNECK, C. A.; SILVA, F. M. B.; BONADIO, I. C.; RIESCO, M. L. G. Resultados maternos e neonatais em Centro de Parto Normal perihospitalar na cidade de São Paulo, Brasil. Revista da Escola de Enfermagem da USP, v. 4 , n. 3, p. 812-818, 2010.

NAGAHAMA, E. E. I.; SANTIAGO, S. M. Práticas de atenção ao parto e os desafios para humanização do cuidado em dois hospitais vinculados ao Sistema Único de Saúde em município da Região Sul do Brasil. Cadernos de Saúde Pública, v. 24, n. 8, p. 18591868, 2008.

NOVO, J. L. V.; PIANTINO, D. G.; FILOGÔNIO, O. S.; NOVO, N. F. Análise de procedimentos assistenciais ao parto normal em primíparas. Revista da Faculdade de Ciências Médicas de Sorocaba, v. 18, n. 1, p. 30-35, 2016.

OLIVEIRA, F. A. M.; LEAL, G. C. G.; WOLFF, L. D. G.; GONÇALVES, L. S. O uso do Escore de Bologna na avaliação da assistência a partos normais em maternidades. Revista Gaúcha de Enfermagem, v. 36, (spe), p. 177-184, 2015.

SANCHO, L. G.; DAIN, S. Avaliação em Saúde e Avaliação Econômica em Saúde: introdução ao debate sobre seus pontos de interseção. Ciência \& Saúde Coletiva, v. 17, n. 3, p. 765-774, 2012. 
SANDIN-BOJÖ, A. K.; HASHIMOTO, M.; KANAL, K.; SUGIURA, Y. Intrapartum care at a tertiary hospital in Cambodia: a survey using the Bologna Score. Midwifery, v. 28, n. 6, e880-5, 2012.

SOUSA, A. M. M.; SOUZA, K. V.; REZENDE, E. M.; MARTINS, E. F.; CAMPOS, D.; LANSKY, S. Práticas na assistência ao parto em maternidades com inserção de enfermeiras obstétricas, em Belo Horizonte, Minas Gerais. Escola Anna Nery Revista de Enfermagem, v. 20, n.2, p. 324-331, 2016.

VARGENS, O. M. D.; SILVA, A. C. V.; PROGIANTI, J. M. Contribuição de enfermeiras obstétricas para consolidação do parto humanizado em maternidades no Rio de JaneiroBrasil. Revista Escola Anna Nery de Enfermagem, v. 21, n.1, e20170015, 2017.

WORLD HEALTH ORGANIZATION. WHO recommendations: intrapartum care for a positive childbirth experience. Geneva: WHO; 2018. 